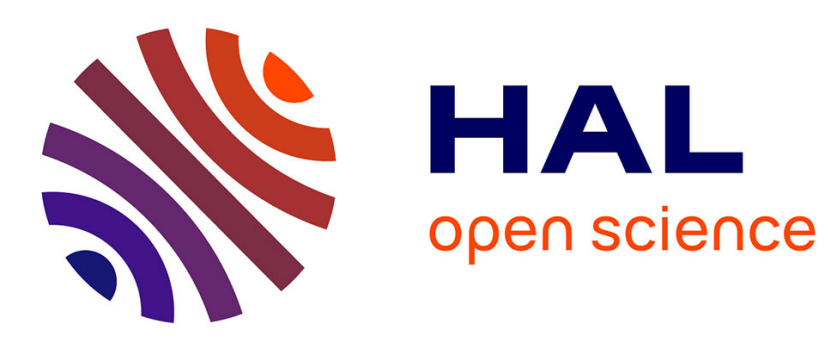

\title{
Stabilization of Linear Systems with Both Input and State Delays by Observer-Predictors
}

Bin Zhou, Qingsong Liu, Frédéric Mazenc

\section{To cite this version:}

Bin Zhou, Qingsong Liu, Frédéric Mazenc. Stabilization of Linear Systems with Both Input and State Delays by Observer-Predictors. Automatica, 2017, 83, pp.368-377. 10.1016/j.automatica.2017.06.027 . hal-01660105

\section{HAL Id: hal-01660105 \\ https://inria.hal.science/hal-01660105}

Submitted on 9 Dec 2017

HAL is a multi-disciplinary open access archive for the deposit and dissemination of scientific research documents, whether they are published or not. The documents may come from teaching and research institutions in France or abroad, or from public or private research centers.
L'archive ouverte pluridisciplinaire HAL, est destinée au dépôt et à la diffusion de documents scientifiques de niveau recherche, publiés ou non, émanant des établissements d'enseignement et de recherche français ou étrangers, des laboratoires publics ou privés. 


\title{
Stabilization of Linear Systems with Both Input and State Delays by Observer-Predictors *
}

\author{
Bin Zhou ${ }^{\mathrm{a}, 1} \quad$ Qingsong Liu $^{\mathrm{a}} \quad$ Frédéric Mazenc $^{\mathrm{b}}$ \\ ${ }^{a}$ Center for Control Theory and Guidance Technology, Harbin Institute of Technology, Harbin, 150001, China. \\ ${ }^{\mathrm{b}}$ EPI DISCO Inria, Laboratoire des Signaux et Systèmes (L2S, UMR CNRS 8506), CNRS CentraleSupélec, Université \\ Paris-Sud, 3 rue Joliot Curie, 91192, Gif-sur-Yvette, France.
}

\begin{abstract}
This paper studies the stabilization of linear systems with both state and input delays where the input delay can be arbitrarily large yet exactly known. Observer-predictor based controllers are designed to predict the future states so that the input delay can be properly compensated. Necessary and sufficient conditions guaranteeing the stability of the closed-loop system are provided in terms of the stability of some simple linear time-delay systems refereed to as observer-error systems, by which the separation principle is discovered. Moreover, approaches in terms of linear matrix inequalities are also provided to design both the state feedback gains and observer gains. Finally, a numerical example illustrates that the proposed approaches are more effective and safe to implement than the existing methods.
\end{abstract}

Key words: Observer-predictor feedback; Time delays; Separation principle; Stabilization.

\section{Introduction}

Control of time-delay systems has received considerable attention and many results have been reported in the literature to deal with various analysis and control problems during the past decades. For example, an improved delay-dependent stability condition for linear systems with time-invariant delays in terms of LMIs was provided in [33], the problem of exponential stability for nonlinear time-delay systems with delayed impulses was addressed in [5], the Lyapunov-Krasovskii functional approach for the stability problem of coupled differentialfunctional equations was discussed in [12], a class of singular systems with time-varying delay was investigated by [6], the problem of networked output tracking control was investigated in [35], sampled-data control modeled as a refined input delayed system was considered in [9], robust stabilization of a system with input delays was investigated in [16], and in [36] the authors proposed two approaches to approximate distributed delay in linear control laws and then to implement it in the $z$-domain

\footnotetext{
* This paper was not presented at any IFAC meeting. The work of B. Zhou was supported in part by the National Natural Science Foundation of China under grant numbers 61322305 and 61573120 , by the Fok Ying Tung Education Foundation under grant number 151060, and by the Foundation for the Author of National Excellent Doctoral Dissertation of China under Grant 201343. The work of F. Mazenc was supported in part by ...

Email addresses: binzhoulee@163.com, binzhou@hit.edu.cn (Bin Zhou), qingsongliu78@163.com (Qingsong Liu),

frederic.mazenc@12s.centralesupelec.fr (Frédéric

Mazenc).

1 Corresponding author
}

and in the $s$-domain.

Predictor feedback is an efficient approach for controlling time-delay systems with input delays and has received renewed interest in recently years. The Smith predictor (SP) was initially proposed in [29] for linear stable systems with an input delay, which, however, may have difficulty in stabilizing unstable systems. Afterwards, a modification of the SP for unstable systems was proposed in [32]. By modelling the input delay dynamic$\mathrm{s}$ as a partial differential equation (PDE) of transport type, the classical predictor feedback for time-delay systems has been re-examined and extended extensively by M. Krstic and his coauthors (see $[17,18]$ for instance). The merit of this approach is that Lyapunov-Krasovskii functionals can be easily constructed and explicit stability estimates can be derived for the closed-loop system. Recently, the predictor feedback was generalized to linear time-varying systems in [25] and a sequential subpredictor was proposed in [27] for linear systems with a long input delay, and extended to some families of timevarying systems in $[19,23,24]$. To the best of our knowledge, the idea of using observers to predict the future state was for the first time introduced in [27] where were considered linear systems with only a single input delay. By safely dropping out the distributed terms in the traditional predictor feedback when the open-loop system is only polynomially unstable, a truncated predictor feedback (TPF) approach was developed in [40] and [39]. The predictor feedback approach has been successfully utilized in [30] to compensate the network-induced time delays for networked control systems.

All the references mentioned above mainly deal with control systems with only input delay. Very recently, 
for linear systems with both input and state delays, the first author of this paper proposed a nested predictor approach to predict the future states such that the input delay that can be arbitrarily large yet bounded is compensated completely [37]. Alternatively approaches by using state transition matrices and by adding integrators to the same problem were proposed respectively in [15] and [38]. The nested predictor feedback has also been extended to neutral-type time-delay systems [41] and discrete-time time-delay systems [20].

In this paper, motivated by the observer based approach in [27] and the predictor feedback approach in [37], we revisit the problem of stabilizing linear systems with both state delay and input delay, which can be arbitrarily large yet exactly known. Our new solutions are based on observer-predictor feedback, which uses both observer and predictor to predict the future states so that the input delay can be properly compensated. We provide necessary and sufficient conditions guaranteeing the stability of the closed-loop system in terms of the stability of some simple linear time-delay systems refereed to as observer-error systems, from which one deduces that the separation principle hods. We also develop approaches in terms of linear matrix inequalities to design both state feedback gains and observer gains in the observer-predictor feedback controllers. The proposed observer-predictor scheme can also be applied on linear systems with only input delay, resulting in controllers that are easy to implement without adding any low-pass filters. We finally use a numerical example to illustrate the effectiveness of the proposed approaches, and, to demonstrate that the proposed approaches are more easy to implement than the existing methods.

This paper is organized as follows: In Section 2, we state the problem to be solved and give some preliminaries. The input delay compensation methods by using a single observer-predictor and multiple observerpredictor are respectively given in Sections 3 and 4. A numerical example is provided to show the effectiveness of the obtained controllers in Section 5. Finally, Section 6 concludes this paper.

\section{Problem Formulation and Preliminaries}

\subsection{Definitions and Notation}

The identity matrix in $\mathbb{R}^{n \times n}$ is denoted by $I_{n}$. The $k \times n$ matrix in which each entry is 0 is denoted by 0 . Given any constant $\tau>0$, we let $C\left([-\tau, 0], \mathbb{R}^{n}\right)$ denote the set of all continuous $\mathbb{R}^{n}$-valued functions defined on $[-\tau, 0]$. We often abbreviate this set (by omitting the dimension which is clear from the context) as $C_{\text {in }}$, and we call it the set of all initial functions. For any continuous function $\varphi:[-\tau, \infty) \rightarrow \mathbb{R}^{n}$ and all $t \geq 0$, we define $\varphi_{t}$ by $\varphi_{t}(m)=\varphi(t+m)$ for all $m \in[-\tau, 0]$, i.e., $\varphi_{t} \in C_{\text {in }}$ is the translation operator. For any matrices $A$ and $B$ in $\mathbb{R}^{n \times n}$, we use $A<B$ to mean that $X^{\top}(B-A) X>0$ for all $X \in \mathbb{R}^{n} \backslash\{0\}$. For two integers $q$ and $q$ with $p \leq q$, let $\mathbf{I}[p, q]=\{p, p+1, \ldots, q\}$.

\subsection{Problem formulation}

We consider a linear time-delay system with both state and input delays as follows

$$
\dot{x}(t)=A x(t)+A_{r} x(t-r)+B u(t-h), \forall t \geq 0,
$$

where $A, A_{r} \in \mathbf{R}^{n \times n}$ and $B \in \mathbf{R}^{n \times m}$ are constant matrices, and $r>0$ and $h>0$ are the state and input delays, respectively. Let the initial condition be $x(\theta)=$ $\varphi(\theta), \forall \theta \in[-r, 0]$, and $u(\theta)=\phi(\theta), \forall \theta \in[-h, 0]$ with $\varphi \in C_{\mathrm{in}}, \phi \in C_{\mathrm{in}}$. Before designing stabilizing controllers for system (1), we first give an assumption as follows.

Assumption 1 The linear time-delay system

$$
\dot{x}(t)=A x(t)+A_{r} x(t-r)+B u(t),
$$

can be stabilized by the following state feedback controller

$$
u(t)=K x(t)+K_{r} x(t-r),
$$

where $K \in \mathbf{R}^{m \times n}$ and $K_{r} \in \mathbf{R}^{m \times n}$ are two gains, namely, the following closed-loop system is asymptotically stable

$$
\dot{x}(t)=(A+B K) x(t)+\left(A_{r}+B K_{r}\right) x(t-r) .
$$

The problem to be solved can be stated as follows.

Problem 1 Under Assumption 1, design a dynamic controller

$$
\left\{\begin{array}{l}
\dot{\chi}(t)=f\left(\chi_{t}, x_{t}, u_{t}\right), \\
u(t)=g\left(\chi_{t}, x_{t}, u_{t}\right),
\end{array}\right.
$$

where $\chi \in \mathbf{R}^{q}$, and $f$ and $g$ are linear functions such that the system (1) in closed-loop with (5) is asymptotically stable.

If $A_{r}=0$, Problem 1 has been studied previously in the literature by using the predictor feedback (see, for example, $[1,17,18,22,27])$. Therefore, in the following, without loss of generality, we assume that $A_{r} \neq 0$.

Under Assumption 1, if the future state $x(t+h)$ is available for feedback, then a solution to Problem 1 is given by

$$
u(t)=K x(t+h)+K_{r} x(t+h-r),
$$

since the closed-loop system is exactly in the form of (4). By the variation of constant formula, the future state $x(t+h)$ can be predicted as

$$
\begin{aligned}
x(t+h)= & \mathrm{e}^{A h} x(t)+\int_{t}^{t+h} \mathrm{e}^{A(t+h-s)} A_{r} x(s-r) \mathrm{d} s \\
& +\int_{t}^{t+h} \mathrm{e}^{A(t+h-s)} B u(s-h) \mathrm{d} s,
\end{aligned}
$$

which is causal (and thus implementable) if and only if $h \leq r$. Hence, without loss of generality, we make the following assumption.

Assumption 2 The input delay $h$ and the state delay $r$ satisfy $h>r$.

Under this assumption, the classical predictor feedback approach $[1,17,18,22]$ does not provide a solution to Problem 1 since the resulting controller is acausal (and is thus not implementable). In order to solve this problem, a nested prediction approach involving multiple integrations was first proposed in [37]. An alternative method by adding integrators was latterly established in [38] to solve the same problem. This problem was also independently studied in [15] by using a completely different method involving the fundamental matrix function for the open-loop time-delay system.

In this paper, based on the first author's early study $[37,38]$ on this problem and motivated by the approach in [27] for stabilization of linear systems with only input 
delays, we will present on observer-predictor based approach to solve Problem 1. Differently from the nested predictor feedback approach [37] and the dynamic compensator method by adding integrators [38], the new approach to be investigated consists in using both observers and predictors to predict the future state $x(t+h)$ firstly and then the observed future state $\widehat{x}(t+h)$ is used in (6) for feedback. The classical separation principle for delayfree linear systems can be extended to this observerpredictor design scheme. To guarantee the stability of the observer-predictor for large input delay $h$, a series of observers with one observer predicting the future state $x\left(t+\Delta_{h}\right)$ with $\Delta_{h}$ small enough are utilized instead.

Dividing large time delays into many small ones to make a difficult design problem solvable or to obtain less conservative results is a valuable idea, and, of course, is not new in the literature. To the best of our knowledge, this idea appeared firstly in Hale's book [14] where it was used to obtain less conservative stability criteria for linear systems with a pure state delay. This delay decomposition approach was then extended by many authors for solving various problems for time-delay systems (see, for example, [13] and [34]). This idea was also used in observer design for nonlinear time-delay systems (see [2] and [10]), feedback control by delayed output [3], and predictor feedback for systems with input delay $[4,27]$.

At the end of this section, we give a brief discussion on the design of the matrices $K$ and $K_{r}$ in Assumption 1. This problem has been studied in [38] (see [38, Lemma $9]$ ), where the method in [13] was adopted. For easy reference, we recall the results in the following lemma.

Lemma 1 [38] There exist two matrices $K$ and $K_{r}$ such that the state feedback controller (3) stabilizes system (2) if there exist symmetric matrices $P>0, Q>0, Z>0$, and two matrices $G$ and $G_{r}$ such that the following matrix inequality is feasible

$$
\left[\begin{array}{ccc}
\Omega_{0} & A_{r} P+B G_{r}+Z & r\left(P A^{\mathrm{T}}+G^{\mathrm{T}} B^{\mathrm{T}}\right) \\
* & -Q-Z & r\left(P A_{r}^{\mathrm{T}}+G_{r}^{\mathrm{T}} B^{\mathrm{T}}\right) \\
* & * & -P Z^{-1} P
\end{array}\right]<0,
$$

where $\Omega_{0}=A P+P A^{\mathrm{T}}+B G+G^{\mathrm{T}} B^{\mathrm{T}}+Q-Z$. Moreover, $K$ and $K_{r}$ are given as $K=G P^{-1}$ and $K_{r}=G_{r} P^{-1}$.

Remark 1 The inequality (8) is not a linear matrix inequality (LMI) due to the term $-P Z^{-1} P$. To overcome this obstacle, we notice that, for any scalar $\alpha>0$, the inequality

$0 \leq(P-\alpha Z) Z^{-1}(P-\alpha Z)=P Z^{-1} P-2 \alpha P+\alpha^{2} Z$, is satisfied, which implies that $-P Z^{-1} P \leq-2 \alpha P+\alpha^{2} Z$. Hence, if the term $-P Z^{-1} P$ is replaced by $-2 \alpha P+\alpha^{2} Z$ in (8), then, for any fixed $\alpha$ and LMI is obtained, which, of course, can then be solved efficiently by the LMI solver in Matlab. The nonlinear term $P Z^{-1} P$ can also be handled efficiently by the so-called Cone Complementarity Linearization Algorithm proposed in [7].

\section{Delay Compensation by A Single Observer- Predictor}

\subsection{Input Delay Compensation by A Single Observer}

In this subsection, a single observer based predictor feedback will be presented to solve Problem 1.
Theorem 1 Let Assumption 1 be satisfied. Consider the following observer based controller

$$
\left\{\begin{aligned}
\dot{\hat{x}}(t)= & A \widehat{x}(t)+A_{r} \widehat{x}(t-r)+B u(t) \\
& +L(\widehat{x}(t-h)-x(t)), \\
u(t)= & K \widehat{x}(t)+K_{r} \widehat{x}(t-r) .
\end{aligned}\right.
$$

Then the closed-loop system consisting of (1) and (9) is asymptotically stable if and only if the following linear time-delay system is asymptotically stable

$$
\dot{e}(t)=A e(t)+A_{r} e(t-r)+L e(t-h) .
$$

Proof. Let

$$
e(t)=\widehat{x}(t-h)-x(t) .
$$

Then, according to (1), (11) and (9), we have

$$
\begin{aligned}
\dot{x}(t)= & A x(t)+A_{r} x(t-r)+B K \widehat{x}(t-h) \\
& +B K_{r} \widehat{x}(t-r-h) \\
= & (A+B K) x(t)+\left(A_{r}+B K_{r}\right) x(t-r) \\
& +B K e(t)+B K_{r} e(t-r),
\end{aligned}
$$

and

$$
\begin{aligned}
\dot{e}(t) & =\dot{\widehat{x}}(t-h)-\dot{x}(t) \\
& =A e(t)+A_{r} e(t-r)+L e(t-h) .
\end{aligned}
$$

The linearity of (12)-(13) implies that the closed-loop system is asymptotically stable if and only if the two systems

$$
\left\{\begin{array}{l}
\dot{\rho}_{1}(t)=(A+B K) \rho_{1}(t)+\left(A_{r}+B K_{r}\right) \rho_{1}(t-r), \\
\dot{\rho}_{2}(t)=A \rho_{2}(t)+A_{r} \rho_{2}(t-r)+L \rho_{2}(t-h),
\end{array}\right.
$$

are. It then follows from Assumption 1 that this is equivalent to the asymptotic stability of system (10). The proof is finished.

From (11) and (13) we can see that the observer state $\widehat{x}(t)$ will approach the system state $x(t+h)$ if the observer-error system (13) or (10) is asymptotically stable, namely, $\widehat{x}(t)$ is an estimation (observer) of $x(t+h)$. Remark 2 Notice that the stability of the observer-error system (10) is independent of the state feedback gains $K$ and $K_{r}$, and is totally determined by the observer gain $L$. This property is quite similar to the separation principle existing in the observer based output feedback design for linear systems without delay [21]. The separation principle indicated by Theorem 1 was not available in [27] where the feedback gains and the observer gains are coupled in the proposed stability conditions.

To verify the stability of the observation error system (10), we present the following proposition whose proof is given in Appendix A1.

Proposition 1 Let $A, A_{r} \in \mathbf{R}^{n \times n}$ be two constant matrices and $r \geq 0$ be a constant.

1. There always exists an observer gain $L$ such that system (10) with $h=0$, namely, the following system

$$
\dot{e}(t)=(A+L) e(t)+A_{r} e(t-r),
$$

is asymptotically stable.

2. Let $L$ be such that system (14) is asymptotically stable, then there exists a constant delay $h^{*}(L)>0$ such that system (10) is also asymptotically stable for all $h \in\left[0, h^{*}(L)\right]$. 
Since $h^{*}(L)$ is expected to be as large as possible, the observer gain $L$ should be carefully designed so that $h^{*}(L)$ is maximized. To this end, we give the following lemma whose proof is straightforward and will be given in Appendix A2 for completeness.

Lemma 2 The observer-error system (10) is asymptotically stable for all $h \in\left[0, h^{*}\right]$, if there exist matrices $P>0, Q>0, Z>0, R>0, S>0$ and a matrix $U$ such that

$$
\left[\begin{array}{ccccc}
\Omega_{1} & \Omega_{2} & 0 & h^{*} P A_{r}^{\mathrm{T}} & r P A_{r}^{\mathrm{T}} \\
* & \Omega_{3} & U+R & -h^{*} P A^{\mathrm{T}} & -r P A^{\mathrm{T}} \\
* & * & -Q-R & -h^{*} U^{\mathrm{T}} & -r U^{\mathrm{T}} \\
* & * & * & -P R^{-1} P & 0 \\
* & * & * & * & -P S^{-1} P
\end{array}\right]<0
$$

is feasible, where $\Omega_{1}=-Z-S, \Omega_{2}=-P A_{r}^{\mathrm{T}}-S, \Omega_{3}=$ $A P+P A^{\mathrm{T}}+Q+Z-S-R$. Moreover, the observer gain can be chosen as $L=U P^{-1}$.

We mention that the two nonlinear terms $P R^{-1} P$ and $P S^{-1} P$ in (15) can be handled by the same method in Remark 1. Now we consider the following optimization problem

$$
h^{\#}=\sup _{P>0, Q>0, Z>0, R>0, S>0, U \in \mathbf{R}^{n \times n}}\left\{h^{*}\right\} \text { s.t. }(15) .
$$

Then it follows from Proposition 1 and Lemma 2 that the observer-error system (10) is asymptotically stable for some $L$ if

$$
h<h^{\#}
$$

\subsection{Input Delay Compensation by A Single Observer- Predictor}

In Theorem 1, the observer is designed to predict the future state $x(t+h)$. However, as indicated by (7), the future state $x(t+r)$ can be obtained by using the predictor

$$
\begin{aligned}
x_{\mathrm{p}}(t+r)= & \mathrm{e}^{A r} x(t)+\int_{t}^{t+r} \mathrm{e}^{A(t+r-s)} A_{r} x(s-r) \mathrm{d} s \\
& +\int_{t}^{t+r} \mathrm{e}^{A(t+r-s)} B u(s-h) \mathrm{d} s .
\end{aligned}
$$

Therefore, by using the predicted state $x_{\mathrm{p}}(t+r)$, one needs only to predict $h-r$ seconds ahead of $x_{\mathrm{p}}(t+r)$. With this idea, we can state the following theorem.

Theorem 2 Let Assumptions 1-2 be satisfied. Consider the following observer-predictor based controller

$$
\left\{\begin{aligned}
\dot{\widehat{x}}(t)= & A \widehat{x}(t)+A_{r} \widehat{x}(t-r)+B u(t) \\
& +L\left(\widehat{x}(t-(h-r))-x_{\mathrm{p}}(t+r)\right), \\
u(t)= & K \widehat{x}(t)+K_{r} \widehat{x}(t-r) .
\end{aligned}\right.
$$

Then the closed-loop system consisting of (1) and (19) is asymptotically stable if and only if the following linear time-delay system is asymptotically stable

$$
\dot{e}(t)=A e(t)+A_{r} e(t-r)+L e(t-(h-r)) .
$$

Proof. According to (1) and (19), the closed-loop system can be rewritten as

$$
\left\{\begin{aligned}
\dot{x}(t)= & A x(t)+A_{r} x(t-r)+B K \widehat{x}(t-h) \\
& +B K_{r} \widehat{x}(t-h-r), \\
\dot{\widehat{x}}(t)= & A \widehat{x}(t)+A_{r} \widehat{x}(t-r)+B K \widehat{x}(t) \\
& +B K_{r} \widehat{x}(t-r)+L(\widehat{x}(t-(h-r)) \\
& -\mathrm{e}^{A r} x(t)-\int_{t}^{t+r} \mathrm{e}^{A(t+r-s)} A_{r} x(s-r) \mathrm{d} s \\
& -\int_{t}^{t+r} \mathrm{e}^{A(t+r-s)} B K \widehat{x}(s-h) \mathrm{d} s \\
& \left.-\int_{t}^{t+r} \mathrm{e}^{A(t+r-s)} B K_{r} \widehat{x}(s-h-r) \mathrm{d} s\right) .
\end{aligned}\right.
$$

We deduce that, for all $t \geq h$,

$$
\begin{aligned}
& \dot{\hat{x}}(t-h)=A \widehat{x}(t-h)+A_{r} \widehat{x}(t-h-r)+B K \widehat{x}(t-h) \\
& +B K_{r} \widehat{x}(t-h-r)+L(\widehat{x}(t-(2 h-r)) \\
& -\mathrm{e}^{A r} x(t-h)-\int_{t-h}^{t-h+r} \mathrm{e}^{A(t-h+r-s)} A_{r} x(s-r) \mathrm{d} s \\
& -\int_{t-h}^{t-h+r} \mathrm{e}^{A(t-h+r-s)} B K \widehat{x}(s-h) \mathrm{d} s \\
& \left.-\int_{t-h}^{t-h+r} \mathrm{e}^{A(t-h+r-s)} B K_{r} \widehat{x}(s-h-r) \mathrm{d} s\right) .
\end{aligned}
$$

Bearing in mind (18) and the fact that $h \geq r$, we deduce that, for all $t \geq h$,

$$
\begin{aligned}
\dot{\widehat{x}}(t-h)= & A \widehat{x}(t-h)+A_{r} \widehat{x}(t-h-r) \\
& +B K \widehat{x}(t-h)+B K_{r} \widehat{x}(t-h-r) \\
& +L(\widehat{x}(t-(2 h-r))-x(t+r-h)) .
\end{aligned}
$$

Let $e(t)=\widehat{x}(t-h)-x(t)$. Then

$\dot{e}(t-h)=A e(t-h)+A_{r} e(t-h-r)+L e(t-(2 h-r))$

and

$$
\begin{aligned}
\dot{x}(t)= & (A+B K) x(t)+\left(A_{r}+B K_{r}\right) x(t-r) \\
& +B K e(t)+B K_{r} e(t-r) .
\end{aligned}
$$

From Assumption 1, we can conclude.

Similarly to the discussion in the above subsection, the observer-error system (20) is asymptotically stable for some $L$ if

$$
h-r<h^{\#} .
$$

The advantage of the observer-predictor based controller (19) is that, when compared with Theorem 1, the stability condition (22) is more easily satisfied than (17).

Remark 3 It has been recognized in the literature that the numerical implementation of the predictor (18) may be unsafe as it can lead to instability of the closed-loop system [26,31,36]. However, the observer-predictor based controller (19) does not suffer such a problem since the observer in (19) is also used as a low-pass filter which helps to avoid the instability problem [26,37]. Moreover, the low-pass filtering property of the observer-filter may help to suppress the measurement noise whenever it exists.

At the end of this subsection, we apply the observerpredictor scheme proposed in Theorem 2 to a linear system with only a single input time delay, as described by

$$
\dot{x}(t)=A x(t)+B u(t-h),
$$

where the system parameters are the same as that in (1) and $(A, B)$ is assumed to be stabilizable. 
Corollary 1 Let $r \in(0, h]$ be any given constant. Consider the following observer-predictor based controller

$$
\left\{\begin{array}{l}
\dot{\hat{x}}(t)=A \widehat{x}(t)+B u(t)+L\left(\widehat{x}(t-(h-r))-x_{\mathrm{p}}(t+r)\right) \\
x_{\mathrm{p}}(t+r)=\mathrm{e}^{A r} x(t)+\int_{t}^{t+r} \mathrm{e}^{A(t+r-s)} B u(s-h) \mathrm{d} s, \\
u(t)=K \widehat{x}(t) .
\end{array}\right.
$$

Then the closed-loop system consisting of (23) and (24) is asymptotically stable if and only if the following linear time-delay system is asymptotically stable

$$
\dot{e}(t)=A e(t)+L e(t-(h-r)) .
$$

Remark 4 The classical predictor feedback for system (23) is

$$
\left\{\begin{aligned}
x(t+h) & =\mathrm{e}^{A h} x(t)+\int_{t}^{t+h} \mathrm{e}^{A(t+h-s)} B u(s-h) \mathrm{d} s, \\
u(t) & =K x(t+h),
\end{aligned}\right.
$$

where $K$ is such that $A+B K$ is Hurwitz. As mentioned in Remark 3, the implementation of the predictor feedback (26) may be unsafe, and to avoid this problem, a low-pass filter is generally required [26]. The essential feature of Corollary 1 is that, differently from the traditional predictor (26), the input delay $h$ is divided into two parts $r$ and $h-r$ with the first part being predicted by the predictor $x_{\mathrm{p}}(t+r)$ and the second parts being predicted by the observer. The most important advantage of this observer-predictor scheme is that, to implement the controller, one does not need to utilize a low-pass filter anymore since the observer itself serves as a low-pass filter, as mentioned by Remark 3. By Proposition 1 we can see that we can always choose a quite large $r$ (such that $h-r$ is sufficiently small) such that the stability of (25) is guaranteed. However, to ensure that the observererror system (25) possesses a fast convergence rate, we may optimize $L$ and $r$ such that the convergence rate of (25) is maximized.

\section{Delay Compensation by Multiple Observer- Predictor}

\subsection{Input Delay Compensation by Multiple Observers}

If $h$ is too large, both the controllers in Theorems 1-2 may fail to stabilize system (1) since the observer-error systems (10) and (20) cannot be stabilized by the choice of an appropriated matrix $L$. Motivated by the approach proposed in [27], we design below a multiple observer based controller to solve Problem 1 . Let $N$ be a positive integer and denote

$$
\Delta_{h}=\frac{h}{N}
$$

Theorem 3 Let Assumptions 1-2 be satisfied. Consider the following observer based controller

$$
\left\{\begin{aligned}
\dot{\widehat{x}}_{i}(t)= & A \widehat{x}_{i}(t)+L_{i}\left(\widehat{x}_{i}\left(t-\Delta_{h}\right)-\widehat{x}_{i+1}(t)\right) \\
& +A_{r} \widehat{x}_{i}(t-r)+B u\left(t-(i-1) \Delta_{h}\right), \\
\dot{\hat{x}}_{N}(t)= & A \widehat{x}_{N}(t)+L_{N}\left(\widehat{x}_{N}\left(t-\Delta_{h}\right)-x(t)\right) \\
& +A_{r} \widehat{x}_{N}(t-r)+B u\left(t-(N-1) \Delta_{h}\right), \\
u(t)= & K \widehat{x}_{1}(t)+K_{r} \widehat{x}_{1}(t-r),
\end{aligned}\right.
$$

where $i \in \mathbf{I}[1, N-1]$ and $\left(K, K_{r}\right)$ satisfies Assumption 1. Then the closed-loop system consisting of (1) and (28) is asymptotically stable if and only if the following series of linear time-delay systems are all asymptotically stable $\dot{e}_{i}(t)=A e_{i}(t)+A_{r} e_{i}(t-r)+L_{i} e_{i}\left(t-\Delta_{h}\right), i \in \mathbf{I}[1, N]$.

Proof. Define a series of prediction error vectors as

$$
\left\{\begin{aligned}
e_{i}(t)= & \widehat{x}_{i}\left(t-(N-i+1) \Delta_{h}\right) \\
& -\widehat{x}_{i+1}\left(t-(N-i) \Delta_{h}\right), i \in \mathbf{I}[1, N-1], \\
e_{N}(t)= & \widehat{x}_{N}\left(t-\Delta_{h}\right)-x(t) .
\end{aligned}\right.
$$

Then one can prove that

$$
\widehat{x}_{1}\left(t-N \Delta_{h}\right)=x(t)+e_{1}(t)+e_{2}(t)+\cdots+e_{N}(t),(30)
$$

for all $t \geq 0$. According to (1) and (28), we have, for all $t \geq 0$,

$$
\begin{aligned}
\dot{x}(t)= & A x(t)+A_{r} x(t-r)+B K \widehat{x}_{1}\left(t-N \Delta_{h}\right) \\
& +B K_{r} \widehat{x}_{1}\left(t-r-N \Delta_{h}\right) \\
= & (A+B K) x(t)+\left(A_{r}+B K_{r}\right) x(t-r) \\
& +B K\left(e_{1}(t)+e(t)+\cdots+e_{N}(t)\right) \\
& +B K_{r}\left(e_{1}(t-r)+e_{2}(t-r)+\cdots+e_{N}(t-r)\right),
\end{aligned}
$$

and

$$
\left\{\begin{aligned}
\dot{e}_{i}(t)= & A e_{i}(t)+A_{r} e_{i}(t-r)+L_{i} e_{i}\left(t-\Delta_{h}\right) \\
& -L_{i+1} e_{i+1}\left(t-\Delta_{h}\right), i \in \mathbf{I}[1, N-1] \\
\dot{e}_{N}(t)= & A e_{N}(t)+A_{r} e_{N}(t-r)+L_{N} e_{N}\left(t-\Delta_{h}\right) .
\end{aligned}\right.
$$

By noting the upper triangular structure of the closedloop system consisting of (31) and (32), we conclude that the closed-loop system is asymptotically stable if and only if

$$
\left\{\begin{array}{l}
\dot{\rho}_{0}(t)=(A+B K) \rho_{0}(t)+\left(A_{r}+B K_{r}\right) \rho_{0}(t-r) \\
\dot{\rho}_{i}(t)=A \rho_{i}(t)+A_{r} \rho_{i}(t-r)+L_{i} \rho_{i}\left(t-\Delta_{h}\right)
\end{array}\right.
$$

is, where $i \in \mathbf{I}[1, N]$. Then it follows from Assumption 1 that this is equivalent to the asymptotic stability of the following system

$$
\dot{e}_{i}(t)=A e_{i}(t)+A_{r} e_{i}(t-r)+L_{i} e_{i}\left(t-\Delta_{h}\right),
$$

where $i \in \mathbf{I}[1, N]$. This allows us to conclude.

We mention that, as pointed out in Remark 2, the separation principle also holds for the observers based controller (28) in Theorem 3. Let $h^{\#}$ be determined by (16). Then the observer-error systems (29) are asymptotically stable for some $L_{i}$ if

$$
\Delta_{h}=\frac{h}{N} \leq h^{\#}
$$

Clearly, the above inequality can be satisfied by choosing $N$ large enough. Since the complexity of the controller (28) is proportional to $N$, one needs to choose the minimal $N^{*}$ such that (34) is satisfied.

Remark 5 Let us denote the convergence rate of system (4) by $\lambda_{K}$ and the convergence rate of the observererror system (29) by $\lambda_{L}$. Then it is not difficult to see that the convergence rate of the closed-loop system consisting of (1) and (28) is $\min \left\{\lambda_{K}, \lambda_{L}\right\}$. The number $\lambda_{K}$ is totally determined by $\left(A, A_{r}, B, h, r\right)$ and can thus be 
considered to be a constant, while $\lambda_{L}$ is determined by $\left\{A, A_{r}, h, r, L_{i}, N\right\}$. Hence we need to design $N$ and $L_{i}$ properly such that $\lambda_{L}$ is maximized. To this end, we let (34) be satisfied and consider:

$$
\widehat{e}_{i}(t)=\mathrm{e}^{\gamma t} e_{i}(t), i \in \mathbf{I}[1, N],
$$

where $\gamma>0$ is a constant. Then the observer-error system (29) can be expressed as

$$
\begin{aligned}
\dot{\hat{e}}_{i}(t)= & \left(A+\gamma I_{n}\right) \widehat{e}_{i}(t)+\mathrm{e}^{\gamma r} A_{r} \widehat{e}_{i}(t-r) \\
& +\mathrm{e}^{\gamma \Delta_{h}} L_{i} \widehat{e}_{i}\left(t-\Delta_{h}\right) \\
\triangleq & A^{\gamma} \widehat{e}_{i}(t)+A_{r}^{\gamma} \widehat{e}_{i}(t-r)+L_{i}^{\gamma} \widehat{e}_{i}\left(t-\Delta_{h}\right),
\end{aligned}
$$

where $i \in \mathbf{I}[1, N]$. Hence, to maximize $\lambda_{L}$, we need only to maximize $\gamma$ subject to the stability of the series of timedelay systems (36) for which Lemma 2 can be used again.

\subsection{Input Delay Compensation by Multiple Observer-} Predictor

In this subsection, we combine the idea in Theorem 2 and the idea in Theorem 3 to propose a multiple observer-predictor based controller to solve Problem 1. Let

$$
\delta_{h}=\frac{h-r}{N} .
$$

Theorem 4 Let Assumptions 1-2 be satisfied. Consider the following observer-predictor based controller

$$
\left\{\begin{aligned}
\dot{\widehat{x}}_{i}(t)= & A \widehat{x}_{i}(t)+A_{r} \widehat{x}_{i}(t-r)+B u\left(t-(i-1) \delta_{h}\right) \\
& +L_{i}\left(\widehat{x}_{i}\left(t-\delta_{h}\right)-\widehat{x}_{i+1}(t)\right), i \in \mathbf{I}[1, N-1], \\
\dot{\widehat{x}}_{N}(t)= & A \widehat{x}_{N}(t)+A_{r} \widehat{x}_{N}(t-r)+B u\left(t-(N-1) \delta_{h}\right) \\
& +L_{N}\left(\widehat{x}_{N}\left(t-\delta_{h}\right)-x_{\mathrm{p}}(t+r)\right), \\
u(t)= & K \widehat{x}_{1}(t)+K_{r} \widehat{x}_{1}(t-r),
\end{aligned}\right.
$$

where $x_{\mathrm{p}}(t+r)$ is determined by (18). Then the closedloop system consisting of (1) and (38) is asymptotically stable if and only if the following linear time-delay systems are all asymptotically stable

$\dot{e}_{i}(t)=A e_{i}(t)+A_{r} e_{i}(t-r)+L_{i} e_{i}\left(t-\delta_{h}\right), i \in \mathbf{I}[1, N]$.

Proof. let us denote the observer-error vectors as $e_{i}(t)=\widehat{x}_{i}\left(t-(N-i+1) \delta_{h}\right)-\widehat{x}_{i+1}\left(t-(N-i) \delta_{h}\right), i \in$ $\mathbf{I}[1, N-1]$ and $e_{N}(t)=\widehat{x}_{N}\left(t-\delta_{h}\right)-x_{\mathrm{p}}(t+r)$. According to (1) and (38), the closed-loop system is

$$
\left\{\begin{aligned}
\dot{x}(t)= & A x(t)+A_{r} x(t-r)+B K \widehat{x}_{1}(t-h) \\
& +B K_{r} \widehat{x}_{1}(t-h-r), \\
\dot{\widehat{x}}_{i}(t)= & A \widehat{x}_{i}(t)+A_{r} \widehat{x}_{i}(t-r)+B K \widehat{x}_{1}\left(t-(i-1) \delta_{h}\right) \\
& +L_{i}\left(\widehat{x}_{i}\left(t-\delta_{h}\right)-\widehat{x}_{i+1}(t)\right) \\
& +B K_{r} \widehat{x}_{1}\left(t-r-(i-1) \delta_{h}\right), i \in \mathbf{I}[1, N-1], \\
\dot{\widehat{x}}_{N}(t)= & A \widehat{x}_{N}(t)+A_{r} \widehat{x}_{N}(t-r)+L_{N} \widehat{x}_{N}\left(t-\delta_{h}\right) \\
& -L_{N}\left(\mathrm{e}^{A r} x(t)+\int_{t}^{t+r} \mathrm{e}^{A(t+r-s)} A_{r} x(s-r) \mathrm{d} s\right. \\
& +\int_{t}^{t+r} \mathrm{e}^{A(t+r-s)} B K \widehat{x}_{1}(s-h) \mathrm{d} s \\
& \left.+\int_{t}^{t+r} \mathrm{e}^{A(t+r-s)} B K_{r} \widehat{x}_{1}(s-h-r) \mathrm{d} s\right) \\
& +B K \widehat{x}_{1}\left(t-(N-1) \delta_{h}\right) \\
& +B K_{r} \widehat{x}_{1}\left(t-r-(N-1) \delta_{h}\right) .
\end{aligned}\right.
$$

Therefore, system (40) in the frequency domain can be rewritten as

$$
\Pi(s)\left[X^{\mathrm{T}}(s), \widehat{X}_{1}^{\mathrm{T}}(s), \cdots, \widehat{X}_{N}^{\mathrm{T}}(s)\right]^{\mathrm{T}}=0,
$$

where $\mathcal{L}(x(t))=X(s), \mathcal{L}\left(\widehat{x}_{i}(t)\right)=\widehat{X}_{i}(s), i \in \mathbf{I}[1, N]$, and

$$
\Pi(s)=\left[\begin{array}{ccccccc}
\Sigma_{0} & \Sigma_{3} & 0 & \cdots & 0 & \cdots & 0 \\
0 & \Lambda_{1}+\Theta_{1} & L_{1} & \cdots & 0 & \cdots & 0 \\
0 & \Theta_{2} & \Lambda_{2} & L_{2} & & \cdots & 0 \\
\vdots & \vdots & & \ddots & \ddots & \ddots & \vdots \\
0 & \Theta_{i} & 0 & & \Lambda_{i} & L_{i} & 0 \\
\vdots & \vdots & \vdots & & \ddots & \ddots & \vdots \\
\Sigma_{2} & \Sigma_{1}+\Theta_{N} & 0 & \cdots & 0 & \cdots & \Lambda_{N}
\end{array}\right]
$$

in which

$$
\left\{\begin{aligned}
\Sigma_{0}= & s I_{n}-A-A_{r} \mathrm{e}^{-s r} \\
\Sigma_{1}= & L_{N}\left(\left(s I_{n}-A\right)^{-1} \mathrm{e}^{-s h}\left(\mathrm{e}^{s r}-\mathrm{e}^{A r}\right) B K\right. \\
& \left.+\left(s I_{n}-A\right)^{-1} \mathrm{e}^{-s h}\left(I_{n}-\mathrm{e}^{-\left(s I_{n}-A\right) r}\right) B K_{r}\right), \\
\Sigma_{2}= & L_{N} \mathrm{e}^{A r}+L_{N}\left(s I_{n}-A\right)^{-1}\left(I_{n}-\mathrm{e}^{-\left(s I_{n}-A\right) r}\right) A_{r}, \\
\Sigma_{3}= & -B K \mathrm{e}^{-h s}-B K_{r} \mathrm{e}^{-(h+r) s} \\
\Theta_{i}= & -B K \mathrm{e}^{-(i-1) \delta_{h} s}-B K_{r} \mathrm{e}^{-r-(i-1) \delta_{h} s}, i \in \mathbf{I}[1, N], \\
\Lambda_{i}= & s I_{n}-A-A_{r} \mathrm{e}^{-s r}-L_{i} \mathrm{e}^{-\delta_{h} s}, i \in \mathbf{I}[1, N] .
\end{aligned}\right.
$$

It follows that the characteristic equation of the closedloop system is $\Delta(s)=\operatorname{det} \Pi(s)$ [14]. By some elementary computation, we get $\Delta(s)=\Delta_{\mathrm{c}}(s) \prod_{i=1}^{N} \Delta_{e_{i}}(s)$, in which

$$
\Delta_{\mathrm{c}}(s)=\left|s I_{n}-(A+B K)-\left(A_{r}+B K_{r}\right) \mathrm{e}^{-s r}\right|,
$$

and

$$
\Delta_{e_{i}}(s)=\left|s I_{n}-A-A_{r} \mathrm{e}^{-s r}-L_{i} \mathrm{e}^{-\delta_{h} s}\right|, i \in \mathbf{I}[1, N],
$$

are respectively the characteristic equations of (4) and (39). Assumption 1 allows us to conclude.

Clearly, the separation principle also holds for the observer-predictor based controller (38) in Theorem 4. Let $h^{\#}$ be the constant defined in (16). Then the observer-error systems (39) are asymptotically stable for some $L_{i}$ if

$$
\delta_{h}=\frac{h-r}{N}<h^{\#} .
$$

Again, the above inequality can be satisfied by choosing $N$ large enough. Moreover, for a given $N$, we can see that $\delta_{h}<\Delta_{h}$. Hence, compared with Theorem 3, the advantage of Theorem 4 is that the stability condition (42) is more easily satisfied than (34).

\section{A Numerical Example}

A numerical example is used in this section to demonstrate the advantages of the proposed observer-predictor based approach. We consider the linearized model of liquid propellant rocket motors previously studied in [37] 

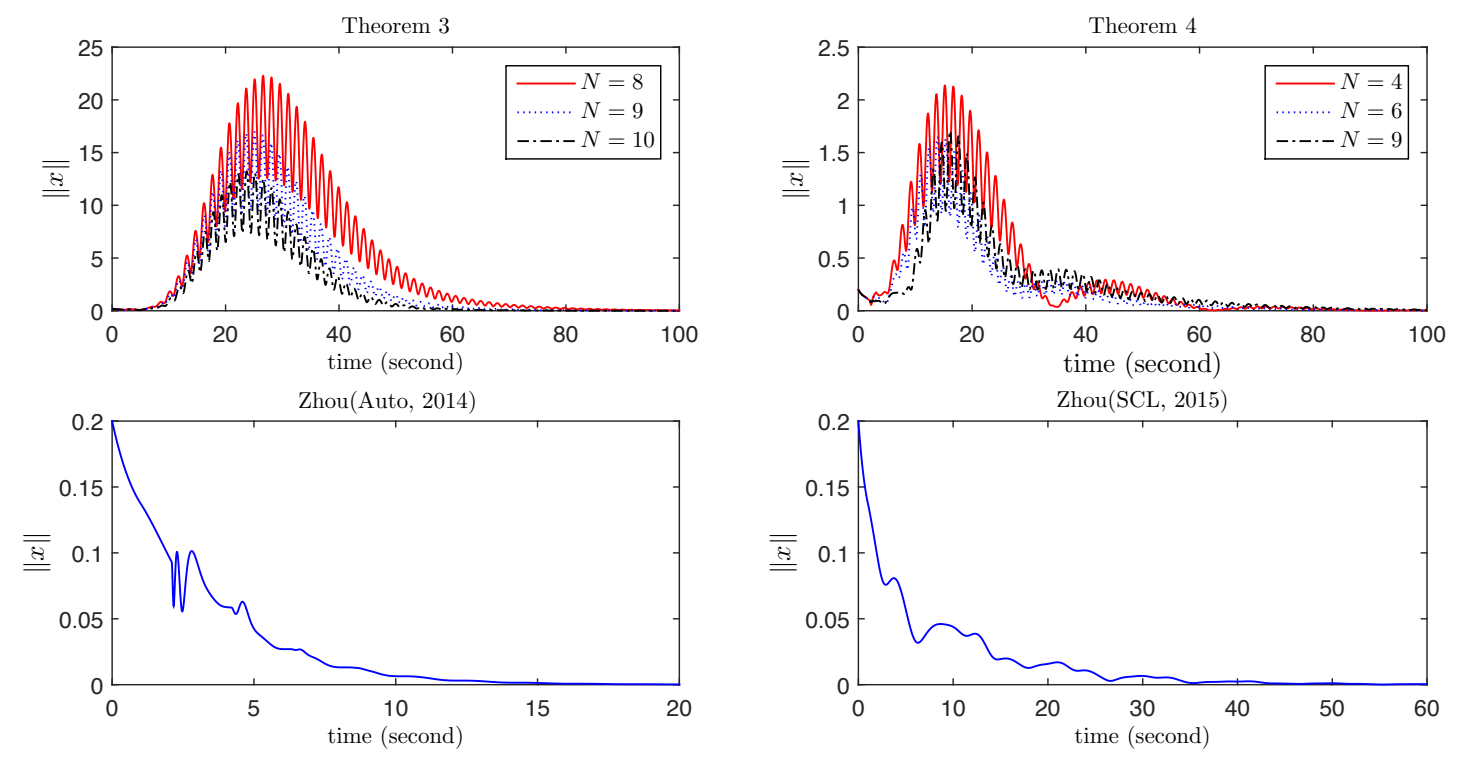

Fig. 1. State trajectories in 2-norm of the closed-loop system consisting of (43) and different controllers.

and [38] as in (1) where

$$
A=\left[\begin{array}{cccc}
0 & 0 & 0 & 0 \\
0 & 0 & 0 & -1 \\
-1 & 0 & -1 & 1 \\
0 & 1 & -1 & 0
\end{array}\right], A_{r}=\left[\begin{array}{cccc}
-1 & 0 & 1 & 0 \\
0 & 0 & 0 & 0 \\
0 & 0 & 0 & 0 \\
0 & 0 & 0 & 0
\end{array}\right], B=\left[\begin{array}{l}
0 \\
1 \\
0 \\
0
\end{array}\right]
$$

where $r=1$ and $h=2.1$. The open-loop system in (43) is unstable due to its two unstable poles at $0.113 \pm i 1.52[8]$. By applying Lemma 1, one can prove that Assumption 1 is fulfilled with

$$
\left\{\begin{array}{l}
K=\left[\begin{array}{llll}
14.0879 & -4.9747 & 5.5178 & -7.4406
\end{array}\right] \\
K_{r}=\left[\begin{array}{llll}
-9.6161 & -0.3932 & 7.6597 & 0.5335
\end{array}\right]
\end{array}\right.
$$

According to Lemma 2, the observer-error system (10) is asymptotically stable if $h<h^{\#}=0.3$. Since $h^{\#}<h$ and $h^{\#}<h-r$, it is unclear (but unlikely) that the controllers provided by Theorems 1 and 2 asymptotically stabilize the system.

We thus need to use the multiple observers based controller (28). Clearly, the inequality (34) is satisfied with $N \geq 8$. For the purpose of comparison, we consider the following three different cases:

i. $N=8$. According to Remark 5, we maximize the scalar $\gamma$ to get $\gamma_{\max }=0.03$ and the corresponding $L_{i}, i \in \mathbf{I}[1,8]$ are given by

$$
L_{i}=\left[\begin{array}{cccc}
-1.4030 & 0.1785 & -0.0314 & 0.0686 \\
-0.0548 & -1.2665 & 0.2052 & 0.8896 \\
0.1533 & 0.4568 & -1.6214 & -0.0119 \\
0.2470 & -0.3028 & 0.9960 & -1.8794
\end{array}\right] \text {. }
$$

ii. $N=9$. According to Remark 5 , we maximize the scalar $\gamma$ to get $\gamma_{\max }=0.06$ and the corresponding
$L_{i}, i \in \mathbf{I}[1,10]$ are given by

$$
L_{i}=\left[\begin{array}{cccc}
-1.5108 & 0.2156 & -0.0925 & 0.1081 \\
-0.0579 & -1.2975 & 0.2060 & 0.9289 \\
0.1431 & 0.5069 & -1.8225 & 0.0179 \\
0.2696 & -0.3068 & 1.0865 & -1.9715
\end{array}\right] \text {. }
$$

iii. $N=10$. According to Remark 5 , we maximize the scalar $\gamma$ to get $\gamma_{\max }=0.08$ and the corresponding $L_{i}, i \in \mathbf{I}[1,10]$ are given by

$$
L_{i}=\left[\begin{array}{cccc}
-1.5903 & 0.2434 & -0.1540 & 0.1527 \\
-0.0238 & -1.3141 & 0.2633 & 0.8442 \\
0.1744 & 0.5240 & -1.9353 & 0.0334 \\
0.2572 & -0.2971 & 1.0993 & -1.9495
\end{array}\right] \text {. }
$$

We can also use the multiple observer-predictor based controller (38) for which the inequality (42) is satisfied with $N \geq 4$. For the purpose of comparison, we consider the following three different cases:

a. $N=4$. According to Remark 5 , we maximize the scalar $\gamma$ to get $\gamma_{\max }=0.02$ and the corresponding $L_{i}, i \in \mathbf{I}[1,4]$ are given by

$$
L_{i}=\left[\begin{array}{cccc}
-1.3624 & 0.1553 & 0.0052 & 0.0375 \\
-0.0908 & -1.2795 & 0.1543 & 0.9686 \\
0.1261 & 0.4454 & -1.5761 & -0.0048 \\
0.2585 & -0.3152 & 0.9961 & -1.9288
\end{array}\right] \text {. }
$$

b. $N=6$. According to Remark 5 , we maximize the scalar $\gamma$ to get $\gamma_{\max }=0.11$ and the corresponding $L_{i}, i \in \mathbf{I}[1,6]$ are given by

$$
L_{i}=\left[\begin{array}{cccc}
-1.7111 & 0.2768 & -0.2159 & 0.1775 \\
-0.0724 & -1.3702 & 0.1465 & 1.0598 \\
0.0862 & 0.5999 & -2.2389 & 0.1021 \\
0.3246 & -0.3315 & 1.3169 & -2.1951
\end{array}\right] \text {. }
$$


Table 1: Computation time for different controllers

\begin{tabular}{c|cc|ccc|ccc}
\hline \hline \multirow{2}{*}{ Different Controllers } & \multicolumn{2}{|c|}{ Existing Methods } & \multicolumn{3}{|c|}{ Theorem 3} & \multicolumn{3}{c}{ Theorem 4} \\
\cline { 2 - 9 } & {$[37]$} & {$[38]$} & $N=8$ & $N=9$ & $N=10$ & $N=4$ & $N=6$ & $N=9$ \\
\hline Computation Time (seconds) & 611 & 26.9 & 3.5 & 4.0 & 4.7 & 64.6 & 69.1 & 81.5 \\
\hline
\end{tabular}

c. $N=9$. According to Remark 5, we maximize the scalar $\gamma$ to get $\gamma_{\max }=0.16$ and the corresponding $L_{i}, i \in \mathbf{I}[1,9]$ are given by

$$
L_{i}=\left[\begin{array}{cccc}
-1.8996 & 0.3356 & -0.3173 & 0.2949 \\
0.0256 & -1.4591 & 0.4033 & 0.7312 \\
0.2548 & 0.6036 & -2.3907 & 0.1707 \\
0.2569 & -0.2790 & 1.2541 & -2.0536
\end{array}\right] \text {. }
$$

Let the initial conditions be $x(\theta)=[0.1,0.1,0.1,0.1]^{\mathrm{T}}$ and $\widehat{x}(\theta)=[0.1,0.1,0.1,0.1]^{\mathrm{T}}, \theta \leq 0$. Then the closedloop systems controlled by the multiple observers based controller (28) and the multiple observer-predictor based controller (38) with different values of $N$ are recorded in Fig. 1. For the sake of comparison, the state trajectories of the closed-loop system controlled by the approaches in [37] and [38] are also recorded in Fig. 1. The computation time (Dell, AMD A4-5300 @ 3.40GHz) required by different approaches are recorded in Table 1 . From these figures and table we can observe the following facts.

- For both the multiple observer-based controller (28) and the multiple observer-predictor based controller (38), larger $N$ can lead to larger convergence rate of the closed-loop system, at the cost of a longer computation time.

- The minimum value of $N$ required by the observer based controller (28) in Theorem 3 is larger than the one required by the observer-predictor based controller (38) in Theorem 4, namely, the advantage of Theorem 4 is that it requires less observers than Theorem 3 .

- The convergence rate of the closed-loop system obtained by applying Theorem 3 with $N=10$ is comparable to that of the closed-loop system obtained by the method in [38]. The computation time required by the method of the present work is shorter than the one of [38].

- Although the convergence rate of the closed-loop system controlled by the method proposed in the present paper is smaller than that in [37], the computation time required by the method of the present work is significantly shorter than that in [37], which shows that the observer based controllers are easier to implement than the one in [37].

To summarize, the proposed observer-based controller and the observer-predictor based controller are respectively easier and safer to implement than those provided by the approaches proposed in the early works [37] and [38], and, in some cases, the control performances are similar.

\section{Conclusion}

This paper investigated the stabilization of linear systems with both state and input delays where the input delay is arbitrarily large yet exactly known. Observer- predictor based controllers were constructed to predict the future states so that the input delay can be compensated properly. Necessary and sufficient conditions guaranteeing the stability of the closed-loop system are provided in terms of the stability of observer-error systems, from which one can deduce that the separation principle holds. Linear matrix inequalities based methods were also used to design feedback gains and observer gains so that the closed-loop system is asymptotically stable. The proposed methods were compared with the existing results in the literature via a numerical example and were found to be more easy to implement than the existing ones.

\section{Appendix}

\section{A1. Proof of Proposition 1}

Proof of Item 1: According to [14] (pp. 106-107), the linear time-delay system (14) is asymptotically stable for any $r \geq 0$ if there exist symmetric matrices $P>0$ and $Q>0$ such that

$$
\left[\begin{array}{cr}
(A+L)^{\mathrm{T}} P+P(A+L)+Q & P A_{r} \\
A_{r}^{\mathrm{T}} P & -Q
\end{array}\right]<0 .
$$

Let $L=-A-l I_{n}$, where $l>0$ is a scalar to be determined. Then, for any given $P>0$ and $Q>0$,

$$
\left[\begin{array}{cc}
-2 l P+Q & P A_{r} \\
A_{r}^{\mathrm{T}} P & -Q
\end{array}\right]<0,
$$

which is always feasible by choosing $l$ sufficiently large.

Proof of Item 2: Let $\Delta_{0}(s)$ and $\Delta_{h}(s)$ denote respectively the characteristic quasi-polynomial of system (14) and system (10), namely,

$$
\Delta_{0}(s)=\operatorname{det}\left(s I_{n}-(A+L)-A_{r} \mathrm{e}^{-r s}\right),
$$

and

$$
\Delta_{h}(s)=\operatorname{det}\left(s I_{n}-A-L \mathrm{e}^{-h s}-A_{r} \mathrm{e}^{-r s}\right) .
$$

It is not hard to rewrite (46) as

$$
\Delta_{h}(s)=\operatorname{det} \Delta_{0}(s) \operatorname{det}\left(I_{n}+\Delta_{0}^{-1}(s) L\left(1-\mathrm{e}^{-h s}\right)\right) .
$$

If

$$
\sup _{\operatorname{Re} s \geq 0}\left\|\Delta_{0}^{-1}(s) L\left(1-\mathrm{e}^{-h s}\right)\right\|<1,
$$

then, for any $s$ satisfying $\operatorname{Re} s \geq 0$, the matrix $I_{n}+$ $\Delta_{0}^{-1}(s) L\left(1-\mathrm{e}^{-h s}\right)$ is nonsingular and thus

$$
\operatorname{det}\left(I_{n}+\Delta_{0}^{-1}(s) L\left(1-\mathrm{e}^{-h s}\right)\right) \neq 0 .
$$

On the other hand, as $\Delta_{0}(s)=0$ has no unstable roots, we know that $\operatorname{det} \Delta_{0}(s) \neq 0$ for any $s$ satisfying $\operatorname{Re} s \geq$ 0 . Therefore, $\Delta_{h}(s) \neq 0$ for any $s$ satisfying Re $s \geq 0$, namely, $\Delta_{h}(s)=0$ has no unstable roots. Notice that

$$
\begin{aligned}
& \sup _{\operatorname{Re} s \geq 0}\left\|\Delta_{0}^{-1}(s) L\left(1-\mathrm{e}^{-h s}\right)\right\| \\
\leq & \sup _{\operatorname{Re} s \geq 0}\left\|\Delta_{0}^{-1}(s) L s\right\| \sup _{\operatorname{Re} s \geq 0}\left|\frac{1-\mathrm{e}^{-h s}}{s}\right| .
\end{aligned}
$$


It is easy to see that

$$
\operatorname{det} \Delta_{0}(s)=p_{0}(n, s)+\sum_{i=1}^{n} p_{i}(n-1, s) \mathrm{e}^{-i r s},
$$

where $p_{0}(n, s)$ and $p_{i}(n-1, s)$ are polynomial functions of $s$ with $\operatorname{deg}\left(p_{0}(n, s)\right)=n$ and $\operatorname{deg}\left(p_{i}(n-1, s)\right) \leq$ $n-1, i \in \mathbf{I}[1, n]$. Note that $\Delta_{0}^{-1}(s)=\frac{\Delta_{0}^{*}(s)}{\operatorname{det} \Delta_{0}(s)}$, where $\Delta_{0}^{*}(s)=\left[\Delta_{0}^{i j}(s)\right]$ is the adjoint matrix of $\Delta_{0}(s)$ with $\operatorname{deg}\left(\Delta_{0}^{i j}(s)\right) \leq n-1, i, j \in \mathbf{I}[1, n]$. Hence, there exists a constant $C(L)$ such that

$$
\sup _{\operatorname{Re} s \geq 0}\left\|\Delta_{0}^{-1}(s) L s\right\| \leq C(L) .
$$

Now, let us prove that

$$
\sup _{\operatorname{Re} s \geq 0}\left|\frac{1-\mathrm{e}^{-s}}{s}\right| \leq 1 .
$$

Let $s=\alpha+\mathrm{i} \beta, \alpha \geq 0$. Then

$$
\sup _{\operatorname{Re} s \geq 0}\left|\frac{1-\mathrm{e}^{-s}}{s}\right|=\sup _{\alpha \geq 0}\left|\frac{1+\mathrm{e}^{-2 \alpha}-2 \mathrm{e}^{-\alpha} \cos \beta}{\alpha^{2}+\beta^{2}}\right|^{\frac{1}{2}} .
$$

Hence, (50) is satisfied if and only if

$$
1+\mathrm{e}^{-2 \alpha} \leq f(\beta),
$$

where $f(\beta)=2 \mathrm{e}^{-\alpha} \cos \beta+\alpha^{2}+\beta^{2}$. Since $f(\beta)$ is an even function and is monotonously increasing for $\beta \in[0, \infty)$, inequality (52) holds true for any real number $\alpha \geq 0$ if $1+\mathrm{e}^{-2 \alpha} \leq f(0)=2 \mathrm{e}^{-\alpha}+\alpha^{2}$, or equivalently $\mathrm{e}^{\alpha}-1 \leq$ $\mathrm{e}^{\alpha} \alpha$. This inequality is true since $\mathrm{e}^{\alpha}-1=\int_{0}^{\alpha} \mathrm{e}(s) \mathrm{d} s \leq$ $\mathrm{e}^{\alpha} \alpha$.

By using inequality (50) we have

$$
\sup _{\operatorname{Re} s \geq 0}\left|\frac{1-\mathrm{e}^{-h s}}{s}\right|=h \sup _{\operatorname{Re} s \geq 0}\left|\frac{1-\mathrm{e}^{-s}}{s}\right| \leq h .
$$

Thus, according to (49) and (53), inequality (47) is satisfied if $h<\frac{1}{C}$. Consequently, any positive number $h^{*}(L)<\frac{1}{C}$ is such that $(10)$ is asymptotically stable for all $h \in\left[0, h^{*}(L)\right]$. This concludes the proof.

\section{A2. Proof of Lemma 2}

The proof is similar to that given in [13]. We choose the following Lyapunov-Krasovskii functional

$$
\begin{aligned}
& V\left(e_{t}\right)=e^{\mathrm{T}}(t) P e(t)+\int_{t-h}^{t} e^{\mathrm{T}}(s) Q e(s) \mathrm{d} s \\
& +\int_{t-r}^{t} e^{\mathrm{T}}(s) Z e(s) \mathrm{d} s+\int_{t-h}^{t} \int_{s}^{t} \dot{e}^{\mathrm{T}}(\tau) R \dot{e}(\tau) \mathrm{d} \tau \mathrm{d} s \\
& +\int_{t-r}^{t} \int_{s}^{t} \dot{e}^{\mathrm{T}}(\tau) S \dot{e}(\tau) \mathrm{d} \tau \mathrm{d} s
\end{aligned}
$$

whose time-derivative can be evaluated as

$$
\begin{aligned}
\dot{V}\left(e_{t}\right)= & 2 e^{\mathrm{T}}(t) P \dot{e}(t)+e^{\mathrm{T}}(t) Q e(t)+e^{\mathrm{T}}(t) Z e(t) \\
& -e^{\mathrm{T}}(t-h) Q e(t-h)-e^{\mathrm{T}}(t-r) Z e(t-r) \\
& +h \dot{e}^{\mathrm{T}}(t) R \dot{e}(t)-\int_{t-h}^{t} \dot{e}^{\mathrm{T}}(s) R \dot{e}(s) \mathrm{d} s \\
& +r \dot{e}^{\mathrm{T}}(t) S \dot{e}(t)-\int_{t-r}^{t} \dot{e}^{\mathrm{T}}(s) S \dot{e}(s) \mathrm{d} s .
\end{aligned}
$$

Applying the Jensen Inequality [11], we can obtain

$$
-\int_{t-h}^{t} \dot{e}^{\mathrm{T}}(s) R \dot{e}(s) \mathrm{d} s \leq-y^{\mathrm{T}}(t) \frac{R}{h} y(t),
$$

and

$$
-\int_{t-r}^{t} \dot{e}^{\mathrm{T}}(s) S \dot{e}(s) \mathrm{d} s \leq-z^{\mathrm{T}}(t) \frac{S}{r} z(t),
$$

where

$$
y(t)=\int_{t-h}^{t} \dot{e}(s) \mathrm{d} s=e(t)-e(t-h),
$$

and

$$
z(t)=\int_{t-r}^{t} \dot{e}(s) \mathrm{d} s=e(t)-e(t-r) .
$$

According to (55) and (56), equation (54) can be rewritten as

$$
\dot{V}\left(e_{t}\right) \leq \eta^{\mathrm{T}}(t) \Omega(h, r) \eta(t)
$$

where

$\eta(t)=\left[\dot{e}^{\mathrm{T}}(t), e^{\mathrm{T}}(t), e^{\mathrm{T}}(t-r), e^{\mathrm{T}}(t-h), y^{\mathrm{T}}(t), z^{\mathrm{T}}(t)\right]^{\mathrm{T}}$, and

$$
\Omega(h, r)=\left[\begin{array}{cccccc}
h R+r S & P & 0 & 0 & 0 & 0 \\
* & Q+Z & 0 & 0 & 0 & 0 \\
* & * & -Z & 0 & 0 & 0 \\
* & * & * & -Q & 0 & 0 \\
* & * & * & * & -\frac{R}{h} & 0 \\
* & * & * & * & * & -\frac{S}{r}
\end{array}\right] .
$$

Moreover, it follows from (10), (57) and (58) that $\Pi \eta(t)=0$ with

$$
\Pi=\left[\begin{array}{cccccc}
I_{n} & -A & -A_{r} & -L & 0 & 0 \\
0 & -I_{n} & 0 & I_{n} & I_{n} & 0 \\
0 & -I_{n} & I_{n} & 0 & 0 & I_{n}
\end{array}\right] .
$$

Therefore, system (10) is asymptotically stable if, for all $\eta(t) \neq 0$ such that $\Pi \eta(t)=0$, there holds $\eta^{\mathrm{T}}(t) \Omega(h, r) \eta(t)<0$. It then follows from the Finsler Lemma [28] that this is equivalent to

$$
\left(\Pi^{\perp}\right)^{\mathrm{T}} \Omega(h, r) \Pi^{\perp}<0,
$$

where $\Pi^{\perp}$ is a right orthogonal complement of $\Pi$, and is given by

$$
\Pi^{\perp}=\left[\begin{array}{cccccc}
-A_{r}^{\mathrm{T}} & 0 & -I_{n} & 0 & 0 & I_{n} \\
A^{\mathrm{T}} & I_{n} & 0 & 0 & I_{n} & I_{n} \\
L^{\mathrm{T}} & 0 & 0 & I_{n} & -I_{n} & 0
\end{array}\right]^{\mathrm{T}} .
$$

Furthermore, applying a Schur complement, (62) is equivalent to

$$
\begin{aligned}
& {\left[\begin{array}{ccc}
-Z & -A_{r}^{\mathrm{T}} P & 0 \\
* & P A+A^{\mathrm{T}} P+Q+Z & P L \\
* & * & -Q
\end{array}\right]} \\
& \quad+h \Pi_{1}^{\mathrm{T}} R \Pi_{1}+r \Pi_{1}^{\mathrm{T}} R \Pi_{1}-\frac{1}{h} \Pi_{2}^{\mathrm{T}} R \Pi_{2}-\frac{1}{r} \Pi_{3}^{\mathrm{T}} S \Pi_{3} \\
& <0,
\end{aligned}
$$

where $\Pi_{1}=\left[A_{r},-A,-L\right], \Pi_{2}=\left[0, I_{n},-I_{n}\right]$, and $\Pi_{3}=$ $\left[I_{n}, I_{n}, 0\right]$. Since $R>0$ and $S>0$, we have $\Omega(h, r) \leq$ $\Omega\left(h^{*}, r\right)$ for all $h$ satisfying $0<h \leq h^{*}$. By applying 
a Schur complement again with $h=h^{*}$, inequality (64) becomes

$$
\left[\begin{array}{ccccc}
\Omega_{1} & \Omega_{4} & 0 & h^{*} A_{r}^{\mathrm{T}} R & r A_{r}^{\mathrm{T}} S \\
* & \Omega_{5} & P L+R & -h^{*} A^{\mathrm{T}} R & -r A^{\mathrm{T}} S \\
* & * & -Q-R & -h^{*} L^{\mathrm{T}} R & -r L^{\mathrm{T}} S \\
* & * & * & -R & 0 \\
* & * & * & * & -S
\end{array}\right]<0,
$$

where $\Omega_{1}=-Z-S, \Omega_{4}=-A_{r}^{\mathrm{T}} P-S, \Omega_{5}=A P+P A^{\mathrm{T}}+$ $Q+Z-S-R$. Multiplying both sides of (65) on the left and on the right by $\operatorname{diag}\left\{P^{-1}, P^{-1}, P^{-1}, R^{-1}, S^{-1}\right\}$ and its transpose, respectively, gives (15), where we have used the substitutions $P^{-1} Z P^{-1} \rightarrow Z, P^{-1} Q P^{-1} \rightarrow$ $Q, P^{-1} \rightarrow P, P^{-1} R P^{-1} \rightarrow R, P^{-1} S P^{-1} \rightarrow S$ and the notation $U=L P$. The proof is finished.

\section{References}

[1] Artstein, Z. (1982). Linear systems with delayed controls: a reduction, IEEE Transactions on Automatic Control, 27(4), 869-879.

[2] Cacace, F., Germania, A. \& Manes, C. (2014). A chain observer for nonlinear systems with multiple timevarying measurement delays, SIAM Journal on Control and Optimization, 52(3), 1862-1885.

[3] Cacace F, Germani A, Manes C. (2014). Predictor-based control of linear systems with large and variable measurement delays, International Journal of Control, 87(4): 704-714.

[4] Cacace, F., Conte, F., Germani, A. \& Pepe, P. (2016). Stabilization of strict-feedback nonlinear systems with input delay using closed-loop predictors, International Journal of Robust and Nonlinear Control, 26, 3524-3540.

[5] Chen, W.-H. \& Zheng, W. X. (2011). Exponential stability of nonlinear time-delay systems with delayed impulse effects, Automatica, 47(5), 1075-1083.

[6] Cong, S. (2014). New stability criteria of linear singular systems with time-varying delay, International Journal of Systems Science, 45(9), 1927-1935.

[7] El Ghaoui, L., Oustry, F. \& AitRami, M. (1997). A cone complementarity linearisation algorithm for static outputfeedback and related problems, IEEE Transactions on Automatic Control, 42, 1171-1176.

[8] Fiagbedzi, Y. A. \& Pearson, A. E. (1986). Feedback stabilization of linear autonomous time lag systems, IEEE Transactions on Automatic Control, 31(9), 847-855.

[9] Fridman, E. (2010). A refined input delay approach to sampled-data control, Automatica, 46(2), 421-427.

[10] Germani, A., Manes, C., \& Pepe, P. (2002). A new approach to state observation of nonlinear systems with delayed output, IEEE Transactions on Automatic Control, 47(1), 96101.

[11] Gu, K., Kharitonov, V. L. \& Chen, J. (2003). Stability of Time-Delay Systems, Berlin: Springer.

[12] Gu, K. \& Liu, Y. (2009). Lyapunov-Krasovskii functional for uniform stability of coupled differential-functional equations, Automatica, 45, 798-804.

[13] Gouaisbaut, F. \& Peaucelle, D. (2006). Delay-dependent robust stability of time delay systems, in the 5th IFAC Symposium on Robust Control Design, Toulouse, France, July 5-7.

[14] Hale, J. K. (1977). Theory of Functional Differential Equations, New York: Springer.

[15] Kharitonov, V. L. (2014). An extension of the prediction scheme to the case of systems with both input and state delay, Automatica, 50(1), 211-217.

[16] Kojima, A., Uchida, K., Shimemura, E. \& Ishijima, S. (1994). Robust stabilization of a system with delays in control, IEEE Transactions on Automatic Control, 39(8), 1694-1698.
[17] Krstic, M. (2009). Delay Compensation for Nonlinear, Adaptive, and PDE Systems. Birkhäuser.

[18] Krstic, M. (2010). Input delay compensation for forward complete and strict-feedforward nonlinear system, IEEE Transactions on Automatic Control, 55(2), 287-303.

[19] Léchappé, V. (2015). Predictive control and estimation of uncertain systems with an input delay, PhD Thesis, Ecole Centrale de Nantes, Nantes, France.

[20] Liu, Q. \& Zhou, B. (2016). Delay compensation of discretetime linear systems by nested prediction, IET Control Theory E6 Applications, 10(15), 1824-1834.

[21] Luenberger, D. G. (1964). Observing the state of a linear system, IEEE Transactions on Military Electronics, 8(2), 7480.

[22] Manitius, A. Z. \& Olbrot, A. W. (1979). Finite spectrum assignment problem for systems with delays, IEEE Transactions on Automatic Control, 24, 541-553.

[23] Mazenc, F. \& Malisoff, M. (2016). Stabilization of nonlinear time-varying systems through a new prediction based approach, IEEE Transactions on Automatic Control. DOI 10.1109/TAC.2016.2600500.

[24] Mazenc, F. \& Malisoff, M. (2016). New prediction approach for stabilizing time-varying systems under time-varying input delay. 55th IEEE Conference Decision and Control, 12-14, December, Las Vegas, USA, 3178-3182.

[25] Mazenc, F., Malisoff, M. \& Niculescu, S.-I. (2014). Reduction model approach for linear time-varying systems with delays, IEEE Transactions on Automatic Control, 59(8), 2068-2082.

[26] Mondié, S. \& Michiels, W. (2003). Finite spectrum assignment of unstable time-delay systems with a safe implementation, IEEE Transactions on Automatic Control, 48, 2207-2212.

[27] Najafi, M., Hosseinniab, S., Sheikholeslama, F. \& Karimadini, M. (2013). Closed-loop control of dead time systems via sequential sub-predictors, International Journal of Control, 86(4), 599-609.

[28] Skelton, R. E., Iwazaki, T. \& Grigoriadis, K. (1998). A Unified Algebraic Approach to Control Design, Taylor and Francis series in Systems and Control.

[29] Smith, O. J. M. (1959). A controller to overcome dead time, ISA Journal, 6(2), 28-33.

[30] Sun, X.-M., Wu, D., Liu, G.-P. \& Wang, W. (2014). Input-to-state stability for networked predictive control with random delays in both feedback and forward channels, IEEE Transactions on Industrial Electronics, 61(7), 3519-3526.

[31] Van Assche, V., Dambrine, M., Lafay, J. F. \& Richard, J. P. (1999). Some problems arising in the implementation of distributed-delay control laws, 38th IEEE Conf. Decision Control, Phoenix, AZ.

[32] Watanabe, K. \& Ito, M. (1981). A process-model control for linear systems with delay, IEEE Transactions on Automatic Control, 26, 1261-1269.

[33] Xu, S. \& Lam, J. (2005). Improved Delay-Dependent Stability Criteria for Time-Delay Systems, IEEE Transactions on Automatic Control, 50, 384-387.

[34] Zhang, X. M. \& Han, Q.-L. (2009). A delay decomposition approach to $H_{\infty}$ control of networked control systems, European Journal of Control, 15, 523-533.

[35] Zhang, J., Lin, Y. \& Shi, P. (2015). Output tracking control of networked control systems via delay compensation controllers, Automatica, 57, 85-92.

[36] Zhong, Q. C. (2004). On distributed delay in linear control laws-Part I: discrete-delay implementations, IEEE Transactions on Automatic Control, 49(11), 2074-2080.

[37] Zhou, B. (2014). Input delay compensation of linear systems with both state and input delays by nested prediction, Automatica, 50(5), 1434-1443.

[38] Zhou, B. (2015). Input delay compensation of linear systems with both state and input delays by adding integrators, Systems \& Control Letters, 82, 51-63. 
[39] Zhou, B. (2014). Truncated Predictor Feedback for Timedelay Systems, XIX, 480 p., Heidelberg, Germany: SpringerVerlag.

[40] Zhou, B., Lin, Z. \& Duan, G. (2012). Truncated predictor feedback for linear systems with long time-varying input delays, Automatica, 48(10), 2387-2399.

[41] Zhou, B. \& Liu, Q. (2017). Input delay compensation for neutral type time-delay systems, Automatica, 78, 309-319. 